\title{
DEGREE OF APPROXIMATION BY POLYNOMIALS TO FUNCTIONS OF BOUNDED VARIATION ${ }^{1}$
}

\author{
HASSOON S. ALIAMIRI
}

1. Introduction. Let $F(z)$ be analytic in the interior of a Jordan curve $C$, continuous in the closed region. For a point $z_{0} \in I(C), F^{\prime}(z)$ is uniquely determined. However, for $z_{0}$ on $C$ we define $F^{\prime}\left(z_{0}\right)$ as $\lim _{z \rightarrow z_{0}}\left(\left(F(z)-F\left(z_{0}\right)\right) /\left(z-z_{0}\right)\right), z$ on $C$ provided this limit exists. Derivatives of higher orders are defined similarly. The subclass of the above functions, where $F^{(p)}(z)$ are of bounded variation on $C$ will be designated throughout as $B(p, C)$. The concept of bounded variation is assumed. Note that if $F \in B(p, C)$, where $C$ is a rectifiable Jordan curve, then $F \in B(q, C), q=0,1, \cdots, p-1$; also every analytic function over a rectifiable Jordan curve is of bounded variation there.

When $C$ is an analytic Jordan curve (analytic), G. Faber [2] proved that analytic functions can be expanded in $I(C)$ by an infinite series of certain polynomials known as Faber polynomials- $\$ 3$.

The object of this paper is to obtain the degree of approximation of such series to functions in $B(p, C)$ (Theorem 1 ). In Lemma $2, \S 2$ an estimate of the Taylor coefficients for functions in $B(p, C)$ where $C$ is $|z|=1$ is obtained. This estimate is employed in obtaining an estimate on the corresponding Faber coefficients in case $C$ is analytic (Lemma 3, §4). Lemma 4, $\$ 5$ gives an estimate on the Faber polynomials associated with the analytic curve $C$. Both Lemmas 4 and 5 are used in proving Theorem 1 . Two related results, Theorem 2 and Theorem 3 , are stated in $\$ 7$ but only the latter is proved.

A similar problem was solved by W. Sewell [4] in which the class of functions was subjected to different hypotheses, mainly that their $p$ th derivatives satisfy a Lipschitz condition of order $\alpha$ on $C, 0<\alpha \leqq 1$.

2. Taylor coefficients. First we mention a Corollary of D. Jackson $[3$, p. 50].

Corollary (JACKSON). Let $F(x)$ be a real-valued function of the real variable $x$ of period $2 \pi$. Let $F^{(p)}(x), p \geqq 0$, exist and be of bounded variation over a period. Then for $k>0$

$$
\left|b_{k}\right| \leqq V\left(F^{(p)}\right) / 2 \pi k^{p+1}, \quad\left|c_{k}\right| \leqq V\left(F^{(p)}\right) / 2 \pi k^{p+1},
$$

Received by the editors March 28, 1966.

1 This paper is part of a doctoral thesis written under the direction of Professors J. Ullman and D. Dickson of the University of Michigan. 
where $b_{k}$ and $c_{k}$ are the kth coefficients of the Fourier series corresponding to $F(x)$ on a period, i.e.,

$$
F(x) \cong b_{0} / 2+\sum_{1}^{\infty}\left(b_{k} \cos k x+c_{k} \sin k x\right) .
$$

$V(G)$ means, throughout, the total variation of $G$.

The above corollary can be extended to include the case where $F(x)$ is a complex-valued function of the real variable $x$. This can be accomplished easily if the above corollary is applied to the real and the imaginary parts of $F(x)$.

LEMмA 1. Let $F(x)$ be a complex-valued function of the real variable $x$ of period $2 \pi$. Let $F^{(p)}(x), p \geqq 0$, exist and be of bounded variation over a period. Then for $k>0$,

$$
\left|b_{k}\right| \leqq V\left(F^{(p)}\right) / \pi k^{p+1}, \quad\left|c_{k}\right| \leqq V\left(F^{(p)}\right) / \pi k^{p+1} .
$$

Lemma 2. Let $F \in B(p, C)$, where $C$ is the circle $|z|=1, p \geqq 0$. Let $G(\theta)=F\left(e^{i \theta}\right)$, and $F(z)=\sum_{0}^{\infty} a_{k} z^{k}$. Then

$$
\left|a_{k}\right| \leqq V\left(G^{(p)}\right) / k^{p+1} \text {. }
$$

Proof. Considering $F\left(e^{i \theta}\right)=G(\theta)$ as a function of $\theta$ it follows that

$$
\begin{aligned}
\frac{b_{k}-i c_{k}}{2} & =\frac{1}{2 \pi i} \int_{0}^{2 \pi} F\left(e^{i \theta}\right)(\cos k \theta-i \sin k \theta) d \theta \\
& =\frac{1}{2 \pi i} \int_{|z|=1} \frac{F(z)}{z^{k+1}} d z=a_{k},
\end{aligned}
$$

where $b_{k}$ and $c_{k}$ are the Fourier coefficients corresponding to $G(\theta)$. Since $F \in B(p, C), C$ is $|z|=1$, then by chain differentiation of $G(\theta)$ $=F\left(e^{i \theta}\right), G^{(p)}(\theta)$ is of bounded variation over $C$. Combining (1) and (3) yields (2).

3. The Faber theorem. Let $C$ be an analytic curve in the $z$-plane and $A(C)$ be its exterior. There is a unique analytic function $f(z)$ which maps $A(C)$ in a 1-1 manner onto the exterior of a circle $|w|=\rho$, designated as $K_{\rho}$, such that the points at infinity correspond to each other and its power series about $z=\infty$ has the normalization $w=f(z)=z+d_{0}+d_{1} / z+\cdots$. Because $f(z)$ is schlicht there exists a unique inverse schlicht function $g(w)$ in $A\left(K_{\rho}\right)$ whose power series expansion about $w=\infty$ is $z=g(w)=w+e_{0}+e_{1} / w+\cdots$, with $\lim \sup \left|e_{n}\right|^{1 / n}=\bar{\rho}, \bar{\rho}<\rho$.

Because of the analyticity of $C$, there is a minimum number $\rho_{0}$, 
$\bar{\rho} \leqq \rho_{0}<\rho$, for which $g(w)$ is still schlicht in $A\left(K_{\rho_{0}}\right)$. Let $C_{\rho_{0}}$ be the level curve in the $z$-plane corresponding to the circle $K_{\rho_{0}}$ under $w=f(z)$. Note that $C$ is $C_{\rho}$. The Faber polynomials $\left\{f_{n}(z)\right\}, n=0,1,2, \cdots$ are the polynomial parts of the formal expansion of $(f(z))^{n}$ about $z=\infty$. In the sequel $f(z), g(w)$ and $f_{n}(z)$ will be used in the above contexts. It is easily seen that

$$
f_{n}(z)=\frac{1}{2 \pi i} \int_{C_{r}} \frac{(f(t))^{n}}{t-z} d t,
$$

where $z \in I\left(C_{r}\right)$ with an appropriate choice $r>\rho_{0}$; see e.g. $[1$, p. 6].

Faber proved the following theorem.

TheOREM (FABER). Let $F(z)$ be analytic in $I(C), C$ an analytic curve. For $z \in I(C)$

$$
F(z)=\sum_{0}^{\infty} a_{k} f_{k}(z)
$$

where $\left\{f_{k}(z)\right\}$ are the Faber polynomials associated with C. Here

$$
a_{k}=\frac{1}{2 \pi i} \int_{K_{\rho_{1}}} \frac{F(g(w))}{w^{k+1}} d w,
$$

for $\rho_{0}<\rho_{1}<\rho$. The series in (5) converges uniformly in any closed subset of $I(C)$.

The coefficients in (5) are referred to as Faber coefficients.

4. Faber coefficients. The following lemma provides an estimate on Faber coefficients.

Lemмa 3. Let $F \in B(p, C), C$ an analytic curve. Then for $k>0, p \geqq 0$,

$$
\left|a_{k}\right|=V\left(G^{(p)}\right) / \rho^{k} k^{p+1}
$$

where $G(\theta)=\sum_{0}^{\infty}\left(a_{k} \rho^{k}\right) e^{i k \theta}, 0 \leqq \theta \leqq 2 \pi$, and $\left\{a_{k}\right\}$ are the Faber coeffcients associated with $F(z)$ in $I\left(C_{\rho}\right)$.

Proof. Since $g(w)$ is schlicht, $F(g(w))$ is analytic in $\rho_{0}<\rho^{\prime} \leqq|w|<\rho$ and continuous in the closure of this annular ring. It is clear from (6) that the Laurent series of $F(g(w))$ in $\rho_{0}<\rho^{\prime} \leqq|w|<\rho$ is

$$
F(g(w))=\sum_{0}^{\infty} a_{k} w^{k}+\sum_{1}^{\infty} A_{-k} / w^{k}
$$

where $a_{k}$ are the Faber coefficients associated with $F(z)$ in $I\left(C_{\rho}\right)$. Let $\alpha(w)=-\sum_{1}^{\infty} A_{-k} / w^{k}, \beta(w)=F(g(w))$. Define 
(9) $\lambda(w)=\alpha(w)+\beta(w)$ for $w$ on $K_{\rho}$ and $\lambda(w)=\sum_{0}^{\infty} a_{k} w^{k}$ for $|w|<\rho$.

Thus $\lambda(w)$ is analytic in $I\left(K_{\rho}\right)$ and continuous in $\bar{I}\left(K_{\rho}\right)$. From (8) and (9) $\lambda(w)=\alpha(w)+\beta(w), \rho^{\prime} \leqq|w| \leqq \rho$. Let $w=\rho y$. Then $\lambda(w)=\lambda(\rho y)$ $=\Phi(y)$ for $|y| \leqq 1$ and

$$
\Phi(y)=\sum_{0}^{\infty}\left(a_{k} \rho^{k}\right) y^{k}, \quad|y|<1 .
$$

By chain differentiation of $\beta(w)$ where $z=g(w)$ it is readily seen that $\beta^{(p)}(w)$ is of bounded variation on $K_{\rho}$. Thus $\lambda \in B\left(p, K_{\rho}\right)$ and since $\Phi^{(p)}(y)=\rho^{p} \lambda^{(p)}(w), \Phi \in B\left(p, K_{1}\right)$. Let $G(\theta)=\Phi\left(e^{i \theta}\right)$ for $0 \leqq \theta \leqq 2 \pi$.

Since $G(\theta)$ is periodic of period $2 \pi$, continuous and of bounded variation over the interval $[0,2 \pi]$, it follows from [6, pp. 175-180] that $G(\theta)=\sum_{0}^{\infty}\left(a_{k} \rho^{k}\right) e^{i k \theta}$. From (2) and (10) it follows $\left|a_{k} \rho^{k}\right|$ $\leqq V\left(G^{(p)}\right) / k^{p+1}$, thus $\left|a_{k}\right| \leqq V\left(G^{(p)}\right) / \rho^{k} k^{p+1}$.

5. Faber polynomials. The following lemma provides an estimate on Faber polynomials.

Lemma 4. Let $C$ be an analytic curve. Then

$$
f_{k}(z)=(f(z))^{k}\left(1+G_{\rho_{1}} \cdot \theta_{k}(z)\right)
$$

for $z \in A\left(C_{\rho_{1}}\right)$ and $\rho_{1}$ a fixed number satisfying $\rho_{1}>\rho_{0} ; G_{\rho_{1}}$ is a real constant independent of $k$ and $z$ but dependent on $\rho_{1}$. The function $\theta_{k}(z)$ is analytic in $\bar{A}\left(C_{\rho_{1}}\right)$ and $\left|\theta_{k}(z)\right| \leqq\left(\rho_{2} / \rho_{1}\right)^{K}$ for $z \in \bar{A}\left(C_{\rho_{1}}\right), k>0$, where $\rho_{2}$ is a definite fixed number with $\rho_{0}<\rho_{2}<\rho_{1}$. Also for $z$ on $C_{\rho^{\prime}}, \rho^{\prime} \geqq \rho_{1}$

$$
\left|f_{k}(z)\right|<\left(1+G_{\rho_{1}}\right) \rho^{\prime k} \text {. }
$$

Proof. Let $\rho_{1}$ be a fixed number such that $\rho_{1}>\rho_{0}$ and let $\rho_{2}$ $=\left(\rho_{1}+\rho_{0}\right) / 2$. For an arbitrary but fixed point $z \in A\left(C_{\rho_{2}}\right)$, choose $\rho_{3}$ large enough, so that $z \in I\left(C_{\rho_{z}}\right)$ and formula (4) holds for $r=\rho_{3}$. Thus

$$
f_{k}(z)=\frac{1}{2 \pi i} \int_{C_{\rho_{3}}} \frac{(f(t))^{k}}{t-z} d t=(f(z))^{k}+\frac{1}{2 \pi i} \int_{C_{\rho_{3}}} \frac{(f(t))^{k}}{t-z} d t .
$$

We put the above in a different form

$$
f_{k}(z)=(f(z))^{k}\left(1+I_{k}(z)\right),
$$

where

$$
I_{k}(z)=\frac{1}{2 \pi i} \int_{C_{\rho_{2}}}\left(\frac{f(t)}{f(z)}\right)^{k} \frac{1}{t-z} d t
$$


Note that the maximum of $\left|I_{k}(z)\right|$ is attained on $C_{\rho_{1}}$ since the function is analytic in the closed exterior of the curve and $\lim _{z \rightarrow \infty} I_{k}(z)=0$. Thus for $z \in \bar{A}\left(C_{\rho_{1}}\right)$,

$$
\max \left|I_{k}(z)\right| \leqq \frac{1}{2 \pi}\left(\frac{\rho_{2}}{\rho_{1}}\right)^{k} \cdot \frac{L}{d\left(C_{\rho_{1}}, C_{\rho_{2}}\right)},
$$

where $L$ is the length of $C_{\rho_{2}}$ and $d\left(C_{\rho_{1}}, C_{\rho_{2}}\right)$ is the minimum distance between $C_{\rho_{1}}$ and $C_{\rho_{2}}$. Let

$$
G_{\rho_{1}}=\frac{L}{2 \pi d\left(C_{\rho_{1}}, C_{\rho_{2}}\right)} \text { and } \theta_{k}(z)=\frac{I_{k}(z)}{G} .
$$

Then formula (11) follows from substituting for $I_{k}(z)$ in the equation of $f_{k}(z)$ above. For $z$ on $C_{\rho^{\prime}}, \rho^{\prime} \geqq \rho_{1}$ it follows that $\left|\theta_{k}(z)\right|$ $\leqq\left(\rho_{2} / \rho_{1}\right)^{k}<1$. Thus formula (12) is proved.

6. The main theorem. Lemmas 3 and 4 yield:

ThEOREM 1. Let $F \in B(p, C)$, where $C$ is an analytic curve and $p \geqq 1$. Then for $z \in \bar{I}(C), n>0$,

$$
\max \left|F(z)-\sum_{0}^{n} a_{k} f_{k}(z)\right| \leqq \frac{Q \cdot V\left(G^{(p)}\right)}{p n^{p}},
$$

where $\sum_{0}^{\infty} a_{k} f_{k}(z)$ is the usual Faber series for $F(z)$ in $I(C)$ and $Q$ is a constant independent of $p, n$ and $z$ but dependent on $\rho$, and $V\left(G^{(p)}\right)$ is the total variation of $G(\theta)$ on $[0,2 \pi]$ and $G(\theta)=\sum_{0}^{\infty}\left(a_{k} \rho^{k}\right) e^{i k \theta}$.

Proof. First we will show that the Faber series converges to $F(z)$ in $\bar{I}(C)$, provided $F \in B(p, C)$, where $C$ is an analytic curve-see $\S 3$. Consider

$$
\left|S_{n+m}(z)-S_{n}(z)\right|=\left|\sum_{n+1}^{n+m} a_{k} f_{k}(z)\right| \leqq \sum_{n+1}^{n+m}\left|a_{k}\right|\left|f_{k}(z)\right|
$$

for $z$ on $C$, where $S_{p}(z)=\sum_{0}^{p} a_{k} f_{k}(z)$. Let $\rho$ be $\rho_{1}$ in Lemma 4. Then (7) and (12) for $z$ on $C$ yield

$$
\begin{aligned}
\max \left|S_{n+m}(z)-S_{n}(z)\right| & \leqq \sum_{k=n+1}^{k=n+m} \frac{V\left(G^{(p)}\right)}{\rho^{k} k^{p+1}}\left(1+G_{\rho}\right) \rho^{k} \\
& \leqq \frac{\left(1+G_{\rho}\right) V\left(G^{(p)}\right)}{\pi} \int_{n}^{\infty} \frac{d u}{u^{p+1}} \\
& =\frac{\left(1+G_{\rho}\right) V\left(G^{(p)}\right)}{\pi p n^{p+1}} .
\end{aligned}
$$


By the maximum principle, for $z \in \bar{I}(C)$,

$$
\left|S_{n+m}(z)-S_{n}(z)\right| \leqq \frac{\left(1+G_{p}\right) V\left(G^{(p)}\right)}{\pi p n^{p+1}} \rightarrow 0, \quad \text { as } n \rightarrow \infty .
$$

By Cauchy's convergence principle $\left\{S_{n}(z)\right\}$ converges uniformly to a function analytic in $I(C)$ and continuous in $\bar{I}(C)$, say $H(z)$. Let $K(z)=F(z)-H(z)$. Since $F(z) \equiv H(z)$ in $I(C)$, it follows that $K(z) \equiv 0$ in $I(C)$ and continuous in $\bar{I}(C)$. Let $z_{0}$ be any fixed point on $C$. Since $C$ is analytic Jordan curve and $K(z)$ is continuous in $\bar{I}(C)$, it follows that $\lim _{z+z_{0}} K(z)=K\left(z_{0}\right)=0$ for $z \in I(C)$. Thus $K(z) \equiv 0$ in $\bar{I}(C)$ which implies $S_{n}(z) \rightarrow F(z)$ uniformly in $\bar{I}(C)$. For $z$ on $C$

$$
\begin{aligned}
\max \left|F(z)-\sum_{0}^{n} a_{k} f_{k}(z)\right| & \leqq \max \sum_{n+1}^{\infty}\left|a_{k}\right| \cdot\left|f_{k}(z)\right| \\
& \leqq \frac{\left(1+G_{\rho}\right) V\left(G^{(p)}\right)}{\pi} \sum_{n+1}^{\infty} \frac{1}{k^{p+1}} \\
& \leqq \frac{\left(1+G_{\rho}\right) V\left(G^{(p)}\right)}{\pi} \int_{n}^{\infty} \frac{d u}{u^{p+1}} \\
& =\frac{Q V\left(G^{(p)}\right)}{p n^{p}},
\end{aligned}
$$

where $Q=\left(1+G_{\rho}\right) / \pi$. By the maximum principle (13) is achieved and so the main theorem is proved.

7. Related results. The method used in proving Theorem 1 can be employed again to show:

Theorem 2. Let $F \in B(p, C)$, where $C$ is an analytic curve and $p \geqq 1$. Let $\sum_{0}^{\infty} a_{k} f_{k}(z)$ be the Faber series of $F(z)$ in $I(C)$. Let $\rho_{1}$, be a fixed number satisfying $\rho_{0}<\rho_{1}<\rho$. Then for $n>0$ and $z \in \bar{I}\left(C_{\rho_{1}}\right)$

$$
\max \left|F(z)-\sum_{0}^{n} a_{k} f_{k}(z)\right|<\frac{Q_{1} V\left(G^{(p)}\right)}{p n^{p}} \cdot\left(\frac{\rho_{1}}{\rho}\right)^{n+1},
$$

where $Q_{1}$ is a constant independent of $p, n$, and $z$ in $I\left(C_{p_{1}}\right)$ but dependent on $\rho_{1}$ and $G(\theta)=\sum_{0}^{\infty}\left(a_{k} \rho^{k}\right) e^{i k \theta}$, for $\theta$ on $[0,2 \pi]$.

ThEOREM 3. Assume the same hypotheses as for the previous theorem. Let $P_{n}(z)$ be the polynomial of degree $n$ found by interpolation to $F(z)$ in the roots of the Faber polynomial $f_{n+1}(z)$. Then for $n$ sufficiently large and $z \in \bar{I}\left(C_{\rho_{1}}\right)$

$$
\max \left|F(z)-P_{n}(z)\right| \leqq \frac{Q_{2} V\left(G^{(p)}\right)}{p n^{p}} \cdot\left(\frac{\rho_{1}}{\rho}\right)^{n+1},
$$


where $Q_{2}$ is a constant independent of $p, n$ and $z$ but dependent on $\rho_{1}$.

The following Theorem due to Sewell and Walsh [5] is needed in the proof of Theorem 3 .

Theorem (SEWell and Walsh). Let $F(z)$ be an analytic function in the interior of an analytic curve $C$ and continuous in its closure. Let $z_{i}, i=1,2, \cdots, n+1$ be in $I(C)$ and $R_{n+1}(z)-\prod^{n+1}\left(z-z_{i}\right)$. Let $P_{n}(z)$ be the polynomial of degree $n$ found by interpolation to $F(z)$ in the points $z_{i}, i=1,2, \cdots, n+1$. Then

$$
F(z)-P_{n}(z)=\frac{1}{2 \pi i} \int_{C} \frac{R_{n+1}(z)}{R_{n+1}(t)} \cdot \frac{F(t)-S_{n}(z)}{t-z} d t,
$$

where $S_{n}(z)$ is an arbitrary polynomial of degree $n$.

Proof of Theorem 3. Let $\rho_{2}=\left(\rho_{1}+\rho_{0}\right) / 2$. The first part of Lemma 4 yields

$$
\max _{z \in \bar{A}(C)}\left|\frac{f_{n+1}(z)}{(f(z))^{n+1}}-1\right| \leqq G_{\rho_{1}}\left(\rho_{2} / \rho_{1}\right)^{n+1}
$$

Thus there exists a positive integer $n_{0}$ such that for $n \geqq n_{0}$

$$
\max _{z \in \bar{A}(C)}\left|\frac{f_{n+1}(z)}{(f(z))^{n+1}}-1\right|<1 / 2,
$$

which implies that for such sufficiently large $n, f_{n+1}(z)$ has no zeros in $\bar{A}(C)$. The Sewell and Walsh Theorem is applicable now when $z_{i}$. $i=1,2, \cdots, n+1$, are chosen to be the zeros of $f_{n+1}(z), n \geqq n_{0}$, $R_{n+1}(z), S_{n}(z)=\sum_{0}^{n} a_{k} f_{k}(z)$. Hence (14) becomes

$$
F(z)-P_{n}(z)=\frac{1}{2 \pi i} \int_{C} \frac{f_{n+1}(z)}{f_{n+1}(t)} \cdot \frac{F(z)-\sum_{0}^{n} a_{k} f_{k}(t)}{t-z} d t
$$

for $z \in I(C)$. For $z$ on $C$ formula (11) yields

$$
\min \left|f_{n+1}(z)\right| \geqq \rho^{n+1}\left|1-G_{\rho_{1}} \theta_{k+1}(z)\right| \geqq \rho^{n+1} / 2
$$

for $n \geqq n_{0}$. The above lower bound and (12) yield

$$
\max \left|\frac{f_{n+1}(z)}{f_{n+1}(t)}\right| \leqq 2\left(1+G_{\rho_{1}}\right)\left(\rho_{1} / \rho\right)^{n+1},
$$

for $n \geqq n_{0}$ where the maximum is taken over $t$ on $C$ and $z$ on $C_{\rho_{1}}$. Formulas (15) and (16) and Theorem 1 yield

$$
\max \left|F(z)-P_{n}(z)\right| \leqq 2\left(1+G_{\rho_{1}}\right)\left(\rho_{1} / \rho\right)^{n+1} \cdot \frac{Q L V\left(G^{(p)}\right)}{2 \pi \delta p n^{p}},
$$


where $L$ is the length of $C, \delta$ is the minimum distance between $C$ and $C_{\rho_{1}}$. Let $Q_{2}=Q L\left(1+G_{\rho_{1}}\right) / \pi \delta$. Then Theorem 3 is concluded.

\section{REFERENCES}

1. H. Al-Amiri, The a-points of Faber polynomials, Bull. College Sci. 8 (1965), 1-25.

2. G. Faber, Über polynomische Entwickelungen, Math. Ann. 57 (1903), 389-408.

3. D. Jackson, The theory of approximation, Amer. Math. Soc. Colloq. Publ. Vol. 11, Amer. Math. Soc., Providence, R. I., 1930.

4. W. Sewell, Degree of approximation by polynomials to continuous functions, Bull. Amer. Math. Soc. 41 (1935), 111-117.

5. W. Sewell and J. Walsh Sufficient conditions for various degrees of approximation by polynomials, Duke Math. J. 6 (1940), 658-705.

6. E. Whittaker and G. Watson, $A$ course of modern analysis, Cambridge University Press, New York, 1950.

Bowling Green State University 